\section{(C) OPEN ACCESS}

\title{
Structural network disruption markers explain disability in multiple sclerosis
}

\author{
Thalis Charalambous, ${ }_{1}^{1}$ Carmen Tur, ${ }^{1}$ Ferran Prados, ${ }^{1,2}$ Baris Kanber, ${ }^{1,2}$ Declan T Chard, ${ }^{1}$ \\ Sebastian Ourselin, ${ }^{2}$ Jonathan D Clayden, ${ }^{3}$ \\ Claudia A M Gandini Wheeler-Kingshott, ${ }^{1,4,5}$ Alan J Thompson, ${ }^{1}$ Ahmed T Toosy ${ }^{1}$
}

\begin{abstract}
- Additional material is published online only. To view please visit the journal online (http://dx.doi.org/10.1136/ jnnp-2018-318440).

${ }^{1}$ Department of Neuroinflammation, UCL Institute of Neurology, Queen Square MS Centre, London, UK ${ }^{2}$ Translational Imaging Group, Centre for Medical Image Computing (CMIC), Department of Medical Physics and Bioengineering, University College London, London, UK ${ }^{3}$ UCL GOS Institute of Child Health, University College London, London, UK

${ }^{4}$ Brain MRI 3T Research Center, C. Mondino National Neurological Institute, Pavia, Italy

${ }^{5}$ Department of Brain and Behavioural Sciences, University of Pavia, Pavia, Italy
\end{abstract}

Correspondence to Thalis Charalambous, Department of Neuroinflammation, UCL Institute of Neurology, Queen Square MS Centre, London WC1B 5EH, UK; thalis. charalambous.09@ucl.ac.uk

Received 16 March 2018 Revised 26 July 2018 Accepted 28 August 2018 Published Online First 22 November 2018

\section{ABSTRACT}

Objective To evaluate whether structural brain network metrics correlate better with clinical impairment and information processing speed in multiple sclerosis (MS) beyond atrophy measures and white matter lesions. Methods This cross-sectional study included 51 healthy controls and 122 patients comprising 58 relapsingremitting, 28 primary progressive and 36 secondary progressive. Structural brain networks were reconstructed from diffusion-weighted MRIs and standard metrics reflecting network density, efficiency and clustering coefficient were derived and compared between subjects' groups. Stepwise linear regression analyses were used to investigate the contribution of network measures that explain clinical disability (Expanded Disability Status Scale (EDSS)) and information processing speed (Symbol Digit Modalities Test (SDMT)) compared with conventional MRI metrics alone and to determine the best statistical model that explains better EDSS and SDMT.

Results Compared with controls, network efficiency and clustering coefficient were reduced in MS while these measures were also reduced in secondary progressive relative to relapsing-remitting patients. Structural network metrics increase the variance explained by the statistical models for clinical and information processing dysfunction. The best model for EDSS showed that reduced network density and global efficiency and increased age were associated with increased clinical disability. The best model for SDMT showed that lower deep grey matter volume, reduced efficiency and male gender were associated with worse information processing speed.

Conclusions Structural topological changes exist between subjects' groups. Network density and global efficiency explained disability above non-network measures, highlighting that network metrics can provide clinically relevant information about MS pathology.

\section{INTRODUCTION}

Multiple sclerosis (MS) is a chronic disease of the central nervous system. Inflammation and demyelination are predominant in relapsing-remitting MS (RRMS), while neurodegeneration is more prominent in the progressive phases (primary progressive MS (PPMS), secondary progressive MS (SPMS)). ${ }^{1}$ Because measures obtained through conventional MRI techniques show incomplete correlation with patients' disability, ${ }^{2}$ more advanced techniques have been used demonstrating that grey matter (GM) atrophy ${ }^{3}$ and abnormalities outside white matter (WM) lesions ${ }^{4}$ also relate to cognitive dysfunction. A very common cognitive domain affected is information processing speed and is assessed by Symbol Digit Modalities Test (SDMT). ${ }^{5}$ Neurological impairment with particular emphasis on ambulation status is evaluated by another widely used measure, the Expanded Disability Status Scale (EDSS). ${ }^{6}$

Brain network analysis has been used to study topological alterations in pathology. ${ }^{7}$ For MS, diffusion-derived networks have shown reduced efficiency correlating with physical disability ${ }^{8}$ and network changes that suggest adaptations to preserve cognitive function. ${ }^{9}$ Whether network metrics explain disability beyond routine imaging metrics is unknown. Only one study addressed this but using only motor network efficiency. ${ }^{10}$ Additionally, network reconstruction techniques have not addressed tractogram biases. ${ }^{11}$ Recent technical work has improved the biological accuracy of streamline tractography, ${ }^{12-14}$ highlighting the necessity of state-of-the-art techniques in network studies. To our knowledge, these techniques have not yet been applied to MS.

In this cross-sectional study, using advanced network reconstruction methods, we aimed (1) to compare structural networks between study subgroups, (2) to investigate whether network metrics explain EDSS and SDMT above conventional MRI metrics, and (3) to determine the best statistical model that explains better EDSS and SDMT.

\section{METHODS}

\section{Participants}

We recruited 122 patients with MS (58 RRMS, 28 PPMS and 36 SPMS) who had not experienced relapses within the preceding 4 weeks and classified as per Lublin and Reingold criteria. ${ }^{15}$ Fifty-one healthy controls (HC) were also examined. Participants underwent MRI and neurological assessment using EDSS. ${ }^{6}$ Verbal SDMT was performed in a subset of MS participants $(n=60)$ (online supplementary etable 1$)$ to screen for information processing speed. Fatigue (visual analogue scale), depression and anxiety (Hospital Anxiety and Depression Scale (HADS)) were also assessed in some patients (online supplementary etable 2). 
MRI data acquisition

MRI data were acquired on a Philips Achieva 3T MR scanner (Philips Healthcare, Best, Netherlands) with a 32-channel head coil using (1) 3D sagittal T1-weighted scans with a fast-field echo scan, (2) whole brain High Angular Resolution Diffusion Imaging scan with echo planar imaging consisted of a cardiac-gated spin-echo sequence and (3) dual-echo proton density/ T2-weighted axial oblique scans. All data were acquired with slices aligned with the anterior commisure - posterior commisure (AC-PC) line to minimise the effect of head positioning on data analysis.

\section{Structural imaging processing}

A non-rigid transformation was performed to register the subject's non-filled T1-weighted bias-field corrected image to the corresponding diffusion-weighted image (DWI) using BrainSuite $\mathrm{V} .15 \mathrm{~b}^{16}$ resulting in a structural image of resolution $2 \times 2 \times 2 \mathrm{~mm}^{3}$. The lesion-filled T1-weighted images ${ }^{17}$ were then segmented into different tissue types and parcellated according to Desikan-Killiany-Tourville atlas protocol using GIF. ${ }^{18}$ The volumes of the various tissue types were estimated (normal-appearing brain volume (NABV), GM, cortical GM (CGM), deep GM (DGM)). Reduction of these volumes reflects atrophy. Lesion load (LL) was also computed as a measure of WM damage.
Diffusion-weighted imaging processing and tractography

The mean b0 image was rigid registered to the first b0 image. Then, the same rigid transformation was applied to the $61 \mathrm{DWI}$ volumes. FSL V.5.0.9 was used on the DWI data to correct for eddy currents and head motion ${ }^{19}$ and BrainSuite V.15b to correct for Echo-planar imaging (EPI) distortions using the T1-weighted image as the registration template for the diffusion data. ${ }^{16}$ For probabilistic tractography, we used second-order integration over fibre orientation distributions (iFOD2) estimated with constrained spherical deconvolution (CSD). ${ }^{20}$ A total of $10^{7}$ streamlines were generated implementing the anatomically constrained tractography (ACT) algorithm ${ }^{12}$ followed by spherical-deconvolution informed filtering of tractograms (SIFT2) ${ }^{13}$ (MRtrix3 V.0.3.14 package).

\section{NETWORK RECONSTRUCTION AND METRICS}

We constructed a symmetric matrix consisting of 120 nodes. Each network edge was defined as the sum of weights of streamlines connecting a pair of nodes. ${ }^{13}$ Figure 1 summarises the pipeline. We extracted a range of standard network measures using TractoR ${ }^{21}$ : Edge density, also known as connectivity, is the ratio of the connections exist relative to the number of potential connections. Global efficiency is a network integration metric that describes the information flow over the entire network while local efficiency is considered a local homolog quantifying

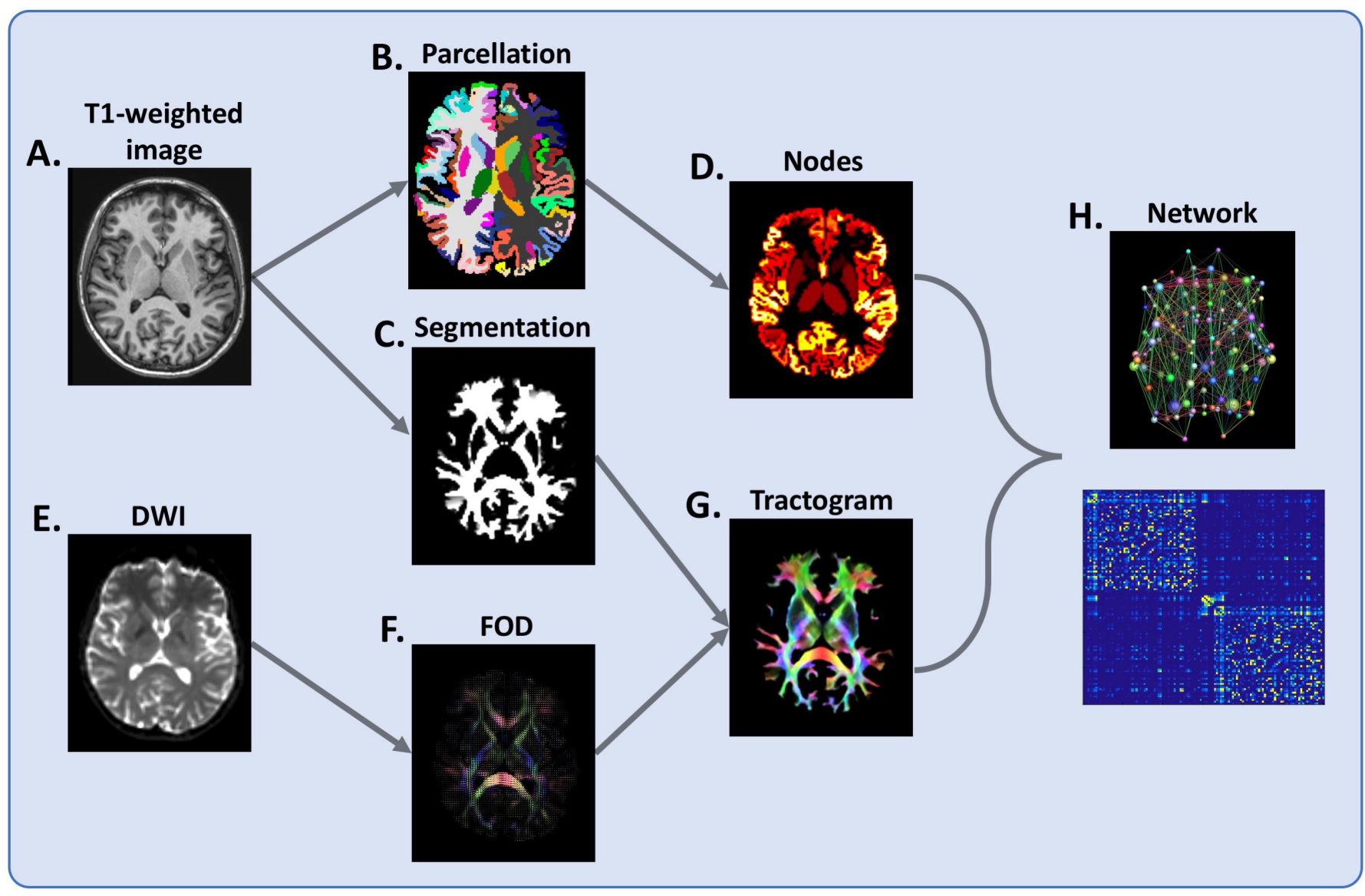

Figure 1 Flowchart of brain network reconstruction. For each subject, (A) T1-weighted image is segmented into grey matter (B) and white matter (C). The grey matter segmentation is parcellated into cortical and deep grey matter regions (B), which serve as network nodes (D) in the subsequent network-based analysis. From a diffusion-weighted image (DWI) (E), voxel-wise fibre orientation distribution (FOD) (F) is estimated and whole-brain tractography undertaken $(\mathrm{G})$, with the white matter segmentation $(\mathrm{C})$ used to prevent this from spilling into grey matter (see main text for details). Finally, nodes and tractogram are modelled into a network $(H)$. Connections are weighted by the sum of the pairwise streamline weights. 


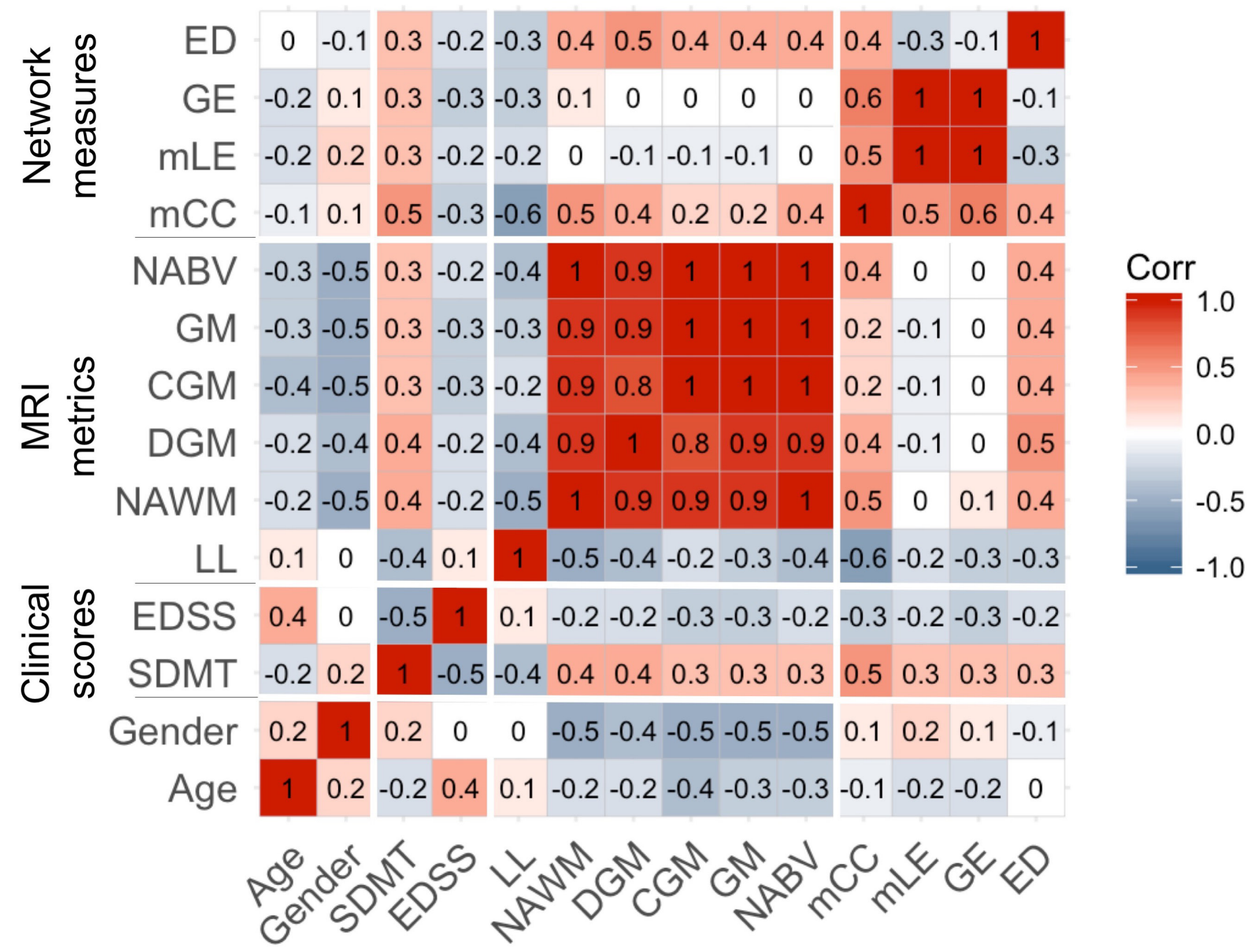

Figure 2 Descriptive pairwise univariable associations in patients. The reported value in each entry of the matrix corresponds to the pairwise Pearson correlation coefficient (r). Gender is a binary variable in which 0 is male and 1 female. CGM, cortical grey matter; DGM, deep grey matter; ED, Edge density; EDSS, Expanded Disability Status Scale; GE, global efficiency; GM, grey matter; LL, lesion load; mCC, mean clustering coefficient; mLE, mean local efficiency; MRI, magnetic resonance imaging; NABV, normal appearing brain volume; NAWM, normal appearing white matter; SDMT, Symbol Digit Modalities Test.

information transfer within local networks. Finally, clustering coefficient reflects the number of connections between neighbouring nodes and is related to network segregation ${ }^{22}$ (for further details on MRI parameters and analysis see online supplementary methods).

\section{Statistical analysis}

Statistical analysis was performed using R software (https://www. r-project.org/ V.3.3.0). For all the models, we explored whether there was a violation of normality assumption of the residuals. Data are reported as mean $\pm S D$, unless otherwise stated. P values $<0.05$ were considered statistically significant.

\section{Preliminary analysis}

To assess network differences between subjects' groups, ANOVA analysis was used, adjusting for age, gender, LL and total intracranial volume (TIV) to correct for head size. To explore possible associations of all the variables in patients, we used bivariate Pearson's correlations. The variables include network metrics (edge density, global efficiency, mean local efficiency and mean clustering coefficient), atrophy measures (NABV, GM,
CGM, DGM), WM damage metrics (LL), clinical scores (EDSS and SDMT) and patient age and gender (figure 2). In this study, atrophy measures and WM lesions are also referred to as MRI metrics. Volumetric differences between HC and patients with MS were also assessed (online supplementary etable 3).

\section{Network measures and volumetric parameters in explaining EDSS and SDMT}

We performed stepwise linear regression analyses using each of the volume metrics (in turn) as independent variables and age, gender and LL as covariates to explain clinical scores (dependent variable). We also controlled for the presence of disease-modifying treatments (DMTs). We selected the best model as assessed with the adjusted $\mathrm{R}^{2}$ (Adj. $\mathrm{R}^{2}$ ) and then added each network metric, in turn, as an independent variable. For SDMT, we performed a post hoc analysis controlling for education level as a categorical and afterwards as a continuous variable to investigate a possible linear relationship between education level and SDMT. To assess whether the effect for each network metric in explaining disability is group dependent, we stratified the MS population based on their clinical profile by creating an 
Table 1 Demographic, clinical, MRI, and network metrics

\begin{tabular}{|c|c|c|c|c|c|}
\hline & $\mathrm{HC}(n=51)$ & $\begin{array}{l}\text { Patients with MS } \\
(n=122)\end{array}$ & RRMS $(n=58)$ & PPMS (n=28) & SPMS $(n=36)$ \\
\hline \multicolumn{6}{|l|}{ Demographics } \\
\hline Age, years & $41 \pm 13$ & $48 \pm 11$ & $42 \pm 10$ & $52 \pm 9$ & $53 \pm 7$ \\
\hline Gender (M/F) & $25 / 26$ & $36 / 86$ & $18 / 40$ & $10 / 18$ & $8 / 28$ \\
\hline Disease duration, years & - & $15 \pm 10$ & $11 \pm 8$ & $14 \pm 7$ & $22 \pm 10$ \\
\hline$\%(n)$ patients of DMTs & - & $58(67)$ & $84(48)$ & $13(3)$ & 47 (16) \\
\hline $\begin{array}{l}\%(n) \text { patients who relapsed in } \\
\text { the previous } 2 \text { years }\end{array}$ & - & $51(38)$ & $68(32)$ & $0(0)$ & $24(6)$ \\
\hline \multicolumn{6}{|l|}{ Clinical scores } \\
\hline EDSS, median & - & $5.5(0-8.5)$ & $2(0-7)$ & $6(3-8)$ & $6.5(4-8.5)$ \\
\hline SDMT & $65.08 \pm 8.31$ & $45.50 \pm 13.27$ & $51.04 \pm 14.28$ & $42.86 \pm 9.46$ & $39.00 \pm 10.88$ \\
\hline \multicolumn{6}{|l|}{ MRI metrics } \\
\hline $\operatorname{NABV}\left(\mathrm{cm}^{3}\right)$ & $1158 \pm 102$ & $1042 \pm 120$ & $1070 \pm 123$ & $1060 \pm 122$ & $984 \pm 93$ \\
\hline $\mathrm{GM}\left(\mathrm{cm}^{3}\right)$ & $679 \pm 57$ & $625 \pm 65$ & $641 \pm 64$ & $632 \pm 67$ & $593 \pm 52$ \\
\hline $\operatorname{CGM}\left(\mathrm{cm}^{3}\right)$ & $640 \pm 54$ & $591 \pm 61$ & $606 \pm 61$ & $597 \pm 65$ & $561 \pm 50$ \\
\hline $\operatorname{DGM}\left(\mathrm{cm}^{3}\right)$ & $39.00 \pm 3.39$ & $34.18 \pm 4.02$ & $34.86 \pm 4.09$ & $35.41 \pm 3.50$ & $32.12 \pm 3.54$ \\
\hline NAWM $\left(\mathrm{cm}^{3}\right)$ & $480 \pm 49$ & $418 \pm 59$ & $429 \pm 62$ & $429 \pm 60$ & $391 \pm 45$ \\
\hline $\mathrm{LL}(\mathrm{mL})$ & - & $14.37 \pm 15.92$ & $12.78 \pm 15.72$ & $16.56 \pm 19.83$ & $15.23 \pm 12.73$ \\
\hline \multicolumn{6}{|l|}{ Network metrics } \\
\hline Edge density, (\%) & $92.6 \pm 2.7$ & $90.6 \pm 3.2$ & $90.8 \pm 3.3$ & $90.5 \pm 3.0$ & $90.3 \pm 3.0$ \\
\hline Global efficiency & $3881 \pm 121$ & $3783 \pm 175$ & $3827 \pm 137$ & $3763 \pm 196$ & $3729 \pm 199$ \\
\hline Mean local efficiency & $3975 \pm 139$ & $3889 \pm 200$ & $3934 \pm 160$ & $3868 \pm 220$ & $3831 \pm 229$ \\
\hline Mean clustering coefficient & $247 \pm 9.2$ & $223 \pm 18.3$ & $227 \pm 17.2$ & $224 \pm 20.5$ & $217 \pm 16.8$ \\
\hline
\end{tabular}

CGM, cortical grey matter; DGM, deep grey matter; DMT, disease-modifying treatment; EDSS, Expanded Disability Status Scale; GM, grey matter; HC, healthy controls; LL, lesion load; MS, multiple sclerosis; NABV, normal-appearing brain volume; NAWM, normal-appearing white matter; PPMS, primary progressive MS; RRMS, relapsing remitting MS; SDMT, Symbol Digit Modalities Test; SPMS, secondary progressive MS.

interaction term, for example the product between the network metric and a categorical variable for MS subgroup (RRMS, PPMS, SPMS), 'network metrics $\times$ MS subgroup', that was then included in the model as an explanatory variable. For SDMT, we explored possible associations between network metrics and MRI variables in $\mathrm{HC}$.

\section{Final models to explain EDSS and SDMT}

To find the best model that explains disability, a stepwise forward selection linear regression strategy was employed. All variables of interest were sequentially added to the model and kept only if significant, culminating in two final models, one per each clinical score.

\section{RESULTS}

Demographic, clinical, MRI and network data from patients with MS and HC are summarised in table 1.

\section{Differences in network metrics in MS population and subtypes}

There was a significant decrease in global efficiency (regression coefficient $(\mathrm{RC})=-71.23, \mathrm{p}=0.016)$, mean local efficiency $(\mathrm{RC}=-72.53, \mathrm{p}=0.031)$ and mean clustering coefficient $(\mathrm{RC}=-14.84, \mathrm{p}<0.0001)$ in the whole MS group when compared with HC. For the subtypes, there was reduced global efficiency in PPMS $(\mathrm{RC}=-85.82, \mathrm{p}=0.027)$ and in SPMS $(\mathrm{RC}=-145.34, \mathrm{p}=0.0002)$ relative to $\mathrm{HC}$ and also decrease in this metric in SPMS relative to RRMS $(\mathrm{RC}=-111.90$, $\mathrm{p}=0.0008$ ). Mean local efficiency was reduced in SPMS compared with $\mathrm{HC}(\mathrm{RC}=-158.42, \mathrm{p}=0.0002)$ and to RRMS $(\mathrm{RC}=-128.21, \mathrm{p}=0.0007)$. Relative to $\mathrm{HC}$, mean clustering coefficient was reduced in RRMS ( $R C=-14.84, \mathrm{p}<0.0001)$, PPMS $(\mathrm{RC}=-13.42, \mathrm{p}<0.0001)$ and SPMS $(\mathrm{RC}=-20.30$, $\mathrm{p}<0.0001)$ while relative to RRMS it was reduced in SPMS $(\mathrm{RC}=-8.30, \mathrm{p}=0.0033)$. There was also a significant decrease in SPMS compared with PPMS $(\mathrm{RC}=-6.88, \mathrm{p}=0.037)$. All models were adjusted for age, gender, LL and TIV (table 2).

\section{Descriptive associations among study variables in patients}

Pairwise associations among clinical, volume and network metrics, LL age and gender study variables are shown in figure 2. Higher LL was associated with lower connectivity $(\mathrm{r}=-0.3)$, lower values of global $(r=-0.3)$ or local $(r=-0.2)$ efficiency and reduced clustering $(\mathrm{r}=-0.6)$. Also, lower connectivity and lower clustering coefficient were associated with reduced volumes of NABV, GM, CGM, DGM and normal-appearing WM (NAWM) ( $\mathrm{r}=$ between 0.2 and 0.5$)$. Moreover, we found associations between clinical scores and network measures; for example, higher EDSS and lower SDMT scores were associated with lower connectivity values, global and local efficiency and clustering coefficient ( $\mathrm{r}=$ between 0.2 and 05). Additionally, correlation analyses between clinical scores and volume metrics demonstrated that higher EDSS scores and lower SDMT were associated with reduced volumes of NABV, GM, CGM, DGM and NAWM $(r=$ between 0.2 and 0.5$)$. Higher LL was also associated with decreased SDMT $(\mathrm{r}=-0.4)$ but showed very little correlation with EDSS score $(r=0.1)$. We also found that higher EDSS score is associated with lower SDMT score $(r=-0.5)$. Gender showed weak associations with network metrics $(r=b e-$ tween -0.1 and 0.2 ). For age, we found that older participants show lower values of network metrics $(r=-0.1$ and 0.2$)$ except edge density that shows weak linear relationship $(r<0.05)$. 
Table 2 Exploratory network differences between different groups

\begin{tabular}{|c|c|c|c|c|c|c|c|c|c|}
\hline & \multicolumn{3}{|l|}{$\mathrm{HC}$} & \multicolumn{3}{|l|}{ RRMS } & \multicolumn{3}{|l|}{ PPMS } \\
\hline & $\mathrm{RC}$ & $95 \% \mathrm{Cl}$ & $P$ values & $\mathrm{RC}$ & $95 \% \mathrm{Cl}$ & $P$ values & $\mathrm{RC}$ & $95 \% \mathrm{Cl}$ & $P$ values \\
\hline \multicolumn{10}{|c|}{ Edge density } \\
\hline MS & -0.65 & $(-1.69$ to 0.38$)$ & 0.210 & & & & & & \\
\hline RRMS & -0.71 & $(-1.84$ to 0.42$)$ & 0.219 & & & & & & \\
\hline PPMS & -0.72 & $(-2.1$ to 0.67$)$ & 0.310 & -0.011 & $(-1.27$ to 1.25$)$ & 0.987 & & & \\
\hline SPMS & -0.45 & $(-1.78$ to 0.88$)$ & 0.507 & 0.258 & $(-0.93$ to 1.43$)$ & 0.670 & 0.27 & $(-1.12$ to 1.66$)$ & 0.707 \\
\hline \multicolumn{10}{|c|}{ Global efficiency } \\
\hline MS & -71.23 & $(-129.47$ to -13.00$)$ & 0.016 & & & & & & \\
\hline RRMS & -33.44 & (-95.11 to 28.25$)$ & 0.287 & & & & & & \\
\hline PPMS & -85.82 & $(-161.6$ to -9.96$)$ & 0.027 & -52.38 & $(-121.08$ to 16.34$)$ & 0.135 & & & \\
\hline SPMS & -145.34 & $(-218.38$ to -72.28$)$ & 0.0001 & -111.90 & $(-176.47$ to -47.31$)$ & 0.0008 & -59.52 & $(-135.72$ to 16.67$)$ & 0.126 \\
\hline \multicolumn{10}{|c|}{ Mean local efficiency } \\
\hline MS & -72.53 & $(-138.60$ to -6.46$)$ & 0.031 & & & & & & \\
\hline RRMS & -30.21 & $(-100.16$ to 39.74$)$ & 0.396 & & & & & & \\
\hline PPMS & -85.68 & $(-171.68$ to 0.34$)$ & 0.051 & -55.46 & (-133.39 to 22.47$)$ & 0.162 & & & \\
\hline SPMS & -158.42 & $(-241.26$ to -75.58$)$ & 0.0002 & -128.21 & (-201.44 to -54.96$)$ & 0.0007 & -72.74 & (-159.16 to 13.68$)$ & 0.099 \\
\hline \multicolumn{10}{|c|}{ Mean clustering coefficient } \\
\hline MS & -14.84 & $(-19.89$ to -9.79$)$ & $<0.0001$ & & & & & & \\
\hline RRMS & -12.00 & (-17.51 to -6.48$)$ & $<0.0001$ & & & & & & \\
\hline PPMS & -13.42 & $(-20.19$ to -6.64$)$ & 0.0001 & -1.42 & $(-7.55$ to 4.73$)$ & 0.650 & & & \\
\hline SPMS & -20.30 & (-26.84 to -13.76$)$ & $<0.0001$ & -8.30 & $(-14.07$ to -2.52$)$ & 0.0033 & -6.88 & $(-13.69$ to -0.06$)$ & 0.037 \\
\hline
\end{tabular}

Analysis of variance was performed. $P$ values in bold denote statistical significance at $p<0.05$ when the groups on the left were compared with the reference group (top row) and adjusted for age, gender, lesion load and total intracranial volume.

HC, healthy controls; MS, multiple sclerosis; PPMS, primary progressive MS; RC, regression coefficient; RRMS, relapsing-remitting MS; SPMS, secondary progressive MS.

\section{Statistical modelling of EDSS score}

We found that NABV was the only significant independent variable of EDSS after adjusting for age, gender and LL. For each millilitre decrease in NABV, there was an increase in the EDSS score of $4.06 \times 10^{-3}$ (95\% CI $-7.68 \times 10^{-3}$ to $-4.3 \times 10^{-3}$, $\mathrm{p}=0.029$, Adj. $\mathrm{R}^{2}=0.185$; table 3$)$. We did not find any significant adjusted associations between the other volume metrics and EDSS.

When network metrics were added to the model reported above as independent variable, in turn, we found that they were each associated with EDSS independently of NABV. Specifically, for each percentage point decrease in edge density, there was an increase in the EDSS score of 0.13 (95\% CI -0.27 to $-1.49 \times 10^{-3}, p=0.047$, Adj. $\left.R^{2}=0.205\right)$, and for each unit decrease in global efficiency, there was an increase in EDSS of $2.67 \times 10^{-3}\left(95 \%\right.$ CI $-4.75 \times 10^{-3}$ to $-5.81 \times 10^{-4}, \mathrm{p}=0.013$, Adj. $\mathrm{R}^{2}=0.221$ ). For each unit decrease in mean local efficiency, there was an increase in EDSS of $1.90 \times 10^{-3}\left(95 \%\right.$ CI $-3.76 \times 10^{-3}$ to $-4.40 \times 10^{-5}, \mathrm{p}=0.045$, Adj. $\mathrm{R}^{2}=0.206$ ), and for each unit decrease in mean clustering coefficient, there was an increase in EDSS of $3.98 \times 10^{-2}\left(95 \% \mathrm{CI}-6.34 \times 10^{-2}\right.$ to $-1.61 \times 10^{-2}$, $\mathrm{p}=0.011$, Adj. $\mathrm{R}^{2}=0.235$ ) (table 3 ). We did not find any significant difference in the effect of any of the network measures in any of the MS subgroups examined. All the above models were adjusted for age, gender, LL and DMTs.

The best model to explain EDSS using the stepwise forward selection linear regression analysis showed that lower edge density, lower global efficiency and increased participants' age explained 26\% of the variance in EDSS (table 3). The explained variance is higher compared with $18.5 \%$ for NABV alone or with $20 \%$ for global efficiency $(-0.02,95 \%$ CI -0.0049 to $-0.00063, p=0.012)$ or with $20 \%$ for edge density $(-0.16$, $95 \%$ CI -0.28 to $-0.035, \mathrm{p}=0.012$ ).

\section{Statistical modelling of SDMT score}

We repeated the multiple linear regression analyses to explain SDMT. When only volume metrics were included, the best model fit was achieved by DGM as independent variable, showing that for each $1 \mathrm{~cm}^{3}$ decrease in the volume of DGM, there was a decrease in the SDMT of 1.61 (95\% CI 0.79 to 2.43 , $\mathrm{p}<0.001$, Adj. $\mathrm{R}^{2}=0.361$; table 4), that is, smaller DGM volumes were associated with worse information processing speed in the whole MS group.

When we added network metrics, in turn, in our multiple regression analysis that included DGM, we found that global efficiency, mean local efficiency and mean clustering coefficient were able to significantly explain additional variance in SDMT. For each unit increase in global efficiency, there was an increase in the SDMT of 0.02 (95\% CI 0.01 to $0.04, p=0.008$, Adj. $\left.R^{2}=0.396\right)$. For each unit increase in mean local efficiency, there was an increase in the SDMT of 0.02 (95\% CI 0.002 to $0.03, \mathrm{p}=0.018$, Adj. $\mathrm{R}^{2}=0.380$ ), and finally for each unit increase in mean clustering coefficient, there was an increase in the SDMT of 0.21 (95\% CI 0.05 to $0.38, p=0.013$, Adj. $\left.R^{2}=0.387\right)$. There was no evidence of change of SDMT per percentage increase in edge density $(0.44$, $95 \%$ CI -0.56 to $1.44, p=0.38$, Adj. $\mathrm{R}^{2}=0.374$; table 1 ). Additionally, there was no significant difference in the effect of any of the network metrics in any of the subgroups examined while the statistical models do not explain SDMT in HC. All the above models were adjusted for age, gender, LL and DMTs.

The best model to explain SDMT using the stepwise forward selection linear regression analysis showed that greater DGM volume, greater global efficiency and female gender were all associated with better information processing speed (table 1). This model explained $39.6 \%$ of the variance in SDMT scores compared with $36 \%$ for the DGM alone $(1.61,95 \%$ CI 0.79 to $2.43, \mathrm{p}<0.001)$. 


\begin{tabular}{|c|c|c|c|c|}
\hline & $\begin{array}{l}\text { Model } \\
\text { summary+predictors }\end{array}$ & $\begin{array}{l}\text { Regression } \\
\text { coefficient }\end{array}$ & $95 \% \mathrm{Cl}$ & $P$ values \\
\hline \multicolumn{5}{|l|}{ MRI metrics } \\
\hline \multirow[t]{4}{*}{ EDSS score } & Adj. $R^{2}=0.185$ & & & \\
\hline & $\mathrm{NABV}, \mathrm{cm}^{3}$ & -0.0041 & $(-0.0077$ to -0.00043$)$ & 0.029 \\
\hline & Age, years & 0.081 & (0.044 to 0.12$)$ & $<0.001$ \\
\hline & Female & -0.73 & $(-1.66$ to 0.20$)$ & 0.125 \\
\hline \multicolumn{5}{|c|}{ MRI metrics+network measures } \\
\hline \multirow[t]{20}{*}{ EDSS score } & Adj. $R^{2}=0.205$ & & & \\
\hline & $\mathrm{NABV}, \mathrm{cm}^{3}$ & -0.0021 & $(-0.0061$ to 0.0019$)$ & 0.297 \\
\hline & Edge density, \% & -0.13 & $(-0.26$ to -0.0014$)$ & 0.047 \\
\hline & Age, years & 0.087 & (0.051 to 0.12 ) & $<0.001$ \\
\hline & Female & -0.60 & $(-1.53$ to 0.33$)$ & 0.202 \\
\hline & Adj. $R^{2}=0.221$ & & & \\
\hline & $\mathrm{NABV}, \mathrm{cm}^{3}$ & -0.0037 & $(-0.0073$ to -0.00016$)$ & 0.041 \\
\hline & Global efficiency & -0.0026 & $(-0.0048$ to -0.00058$)$ & 0.013 \\
\hline & Age, years & 0.072 & (0.036 to 0.11$)$ & $<0.001$ \\
\hline & Female & -0.52 & $(-1.44$ to 0.40$)$ & 0.266 \\
\hline & Adj. $R^{2}=0.206$ & & & \\
\hline & $\mathrm{NABV}, \mathrm{cm}^{3}$ & -0.0041 & $(-0.076$ to -0.00049$)$ & 0.026 \\
\hline & $\mathrm{mLE}$ & -0.0019 & $(-0.0038$ to -0.000044$)$ & 0.045 \\
\hline & Age, years & 0.073 & (0.036 to 0.11$)$ & $<0.001$ \\
\hline & Female & -0.57 & $(-1.50$ to 0.37$)$ & 0.231 \\
\hline & Adj. $R^{2}=0.229$ & & & \\
\hline & $\mathrm{NABV}, \mathrm{cm}^{3}$ & -0.0016 & $(-0.005$ to 0.007$)$ & 0.551 \\
\hline & $\mathrm{mCC}$ & -0.029 & $(-0.051$ to -0.0075$)$ & 0.008 \\
\hline & Age, years & 0.078 & $(-0.0042$ to 0.0022$)$ & $<0.001$ \\
\hline & Female & -0.30 & $(-1.26$ to 0.66$)$ & 0.534 \\
\hline \multicolumn{5}{|l|}{ Final model } \\
\hline \multirow[t]{4}{*}{ EDSS score } & Adj. $R^{2}=0.259$ & & & \\
\hline & Edge density, \% & -0.17 & $(-0.28$ to -0.060$)$ & 0.003 \\
\hline & Global efficiency & -0.0031 & $(-0.0051$ to -0.0011$)$ & 0.003 \\
\hline & Age, years & 0.081 & (0.047 to 0.12$)$ & $<0.001$ \\
\hline
\end{tabular}

$P$ values in bold denote statistical significance at $p<0.05$.

EDSS, Expanded Disability Status Scale; NABV, normal-appearing brain volume; mCC, mean clustering coefficient; $\mathrm{mLE}$, mean local efficiency.

\section{DISCUSSION}

This study showed structural network topological changes within the various MS groups. We also demonstrated that markers of structural network disruption explain EDSS and SDMT scores above metrics of tissue atrophy and WM lesions.

\section{Structural network differences between subjects' groups}

We detected network topological changes in MS. Relative to HC, SPMS had reduced global and local efficiency, PPMS reduced global efficiency while there was no efficiency change in RRMS. These changes reflect network alterations due to diffuse WM pathology including impaired long-distance and short-distance connections, characteristics that are more prominent in the progressive types. Previous studies focused mainly on RRMS reporting decreases in this metric in structural ${ }^{82122}$ and functiona ${ }^{22-24}$ networks while others, in accordance with this work, found no differences. ${ }^{25}$ Intriguingly, increased efficiency in RRMS in the first year from onset in the absence of clinical impairment is suggestive of structural adaptations to maintain normal function. ${ }^{9}$ Our RRMS cohort has a relatively long disease duration with high EDSS due to accrual of baseline disability as a result of incomplete recovery from relapses explaining partly the absence of this effect. Yet, only one study considered SPMS and

\begin{tabular}{|c|c|c|c|c|}
\hline & $\begin{array}{l}\text { Model } \\
\text { summary+predictors }\end{array}$ & $\begin{array}{l}\text { Regression } \\
\text { coefficient }\end{array}$ & $95 \% \mathrm{Cl}$ & $P$ values \\
\hline \multicolumn{5}{|l|}{ MRI metrics } \\
\hline \multirow[t]{4}{*}{ SDMT score } & Adj. $R^{2}=0.361$ & & & \\
\hline & $\mathrm{DGM}, \mathrm{cm}^{3}$ & 1.61 & (0.79 to 2.43 ) & $<0.001$ \\
\hline & Lesion load, mL & -0.17 & $(-0.34$ to -0.0014$)$ & 0.048 \\
\hline & Female & 12.16 & (5.51 to 18.82) & $<0.001$ \\
\hline \multicolumn{5}{|c|}{ MRI metrics+network measures } \\
\hline \multirow[t]{17}{*}{ SDMT score } & Adj. $R^{2}=0.352$ & & & \\
\hline & $\mathrm{DGM}, \mathrm{cm}^{3}$ & 1.52 & (0.61 to 2.43 ) & 0.001 \\
\hline & Lesion load, mL & -0.17 & $(-0.34$ to 0.0069$)$ & 0.059 \\
\hline & Edge density, (\%) & 0.24 & $(-0.75$ to 1.23$)$ & 0.624 \\
\hline & Female & 11.94 & (5.18 to 18.70 ) & $<0.001$ \\
\hline & Adj. $R^{2}=0.396$ & & & \\
\hline & DGM, $\mathrm{cm}^{3}$ & 1.93 & (1.21 to 2.65$)$ & $<0.001$ \\
\hline & Global efficiency & 0.021 & (0.0055 to 0.035$)$ & 0.008 \\
\hline & Female & 10.97 & (4.37 to 17.56 ) & 0.002 \\
\hline & Adj. $R^{2}=0.380$ & & & \\
\hline & DGM, $\mathrm{cm}^{3}$ & 2.01 & (1.28 to 2.75$)$ & $<0.001$ \\
\hline & $\mathrm{mLE}$ & 0.015 & (0.0028 to 0.028$)$ & 0.018 \\
\hline & Female & 11.43 & (4.79 to 18.06 ) & 0.001 \\
\hline & Adj. $R^{2}=0.387$ & & & \\
\hline & $\mathrm{DGM}, \mathrm{cm}^{3}$ & 1.45 & (0.63 to 2.28 ) & $<0.001$ \\
\hline & $\mathrm{mCC}$ & 0.21 & (0.047 to 0.38 ) & 0.013 \\
\hline & Female & 9.92 & (2.98 to 16.85 ) & 0.006 \\
\hline \multicolumn{5}{|l|}{ Final model } \\
\hline \multirow[t]{4}{*}{ SDMT score } & Adj. $R^{2}=0.396$ & & & \\
\hline & $\mathrm{DGM}, \mathrm{cm}^{3}$ & 1.93 & (1.21 to 2.65 ) & $<0.001$ \\
\hline & Global efficiency & 0.021 & $(0.0055$ to 0.035$)$ & 0.008 \\
\hline & Female & 10.97 & (4.36 to 17.56 ) & 0.002 \\
\hline
\end{tabular}

$P$ values in bold denote statistical significance at $p<0.05$.

DGM, deep grey matter; SDMT, Symbol Digit Modalities Test; mCC, mean clustering coefficient; $\mathrm{mLE}$, mean local efficiency.

PPMS group reporting reduced global efficiency, in accordance with our findings. ${ }^{21}$ Moreover, we demonstrate reduced global and local efficiency in SPMS relative to RRMS, a result likely to reflect the neurodegenerative component in this progressive subtype.

Clustering coefficient is a 'small-world' metric and reduction suggests a more random architecture ${ }^{26}$ related to increased disability as shown in our study and elsewhere. ${ }^{27}$ Previous structural studies reported increased clustering coefficient in RRMS compared with $\mathrm{HC}^{9} 2829$ and is thought to reflect transient compensatory changes. No change was reported in functional networks. ${ }^{23}{ }^{30}$ Here, we report a decrease in clustering coefficient in RRMS compared with HC, in agreement with a study that investigated both structural and functional networks. ${ }^{22}$ We also extend these findings demonstrating reduction of this metric in the progressive phases. Clustering coefficient was further reduced in SPMS relative to RRMS and PPMS indicating that impaired local information flow is linked to the disease severity. Nonetheless, further investigations with bigger sample sizes and longitudinal study design should confirm the study findings.

\section{Network measures explain additional variance of disability} Whole brain atrophy is a relatively strong predictor of EDSS. Our study shows that the addition of network metrics into the model, singly and together, explains more EDSS variance, leading to our final model (table 3), according to which edge 
density and global efficiency explain $26 \%$ of the variance, that is 7\% more compared with NABV alone (19\%). Loss of connectivity could reflect neurodegeneration due to continuous inflammation ${ }^{31}$ while reduced global efficiency could indicate impaired structural long-range connections probably due to inflammatory activity and neuroaxonal loss. ${ }^{32}$ The fact that these measures integrate information beyond local tissue damage and atrophy measures may account for the increased explained variance.

SDMT was most strongly associated with DGM atrophy and WM damage. ${ }^{33}$ Previous structural and functional studies demonstrated the relationship between network disruption and cognitive impairment. ${ }^{4334}$ Our study findings showed that global efficiency is associated with SDMT as previously shown and it also explained additional variance (table 1) highlighting that intact network integration is important for efficient information processing beyond participant's education level and treatment. These findings are also consistent across WM diseases ${ }^{35}$ signifying the relevance of network efficiency as potential marker of cognitive disability.

MS is a heterogeneous disease. This study included patients with MS with the main disease phenotypes in order to provide a representative snapshot of structural networks throughout the entire disease course. Our regression analyses show that the behaviour of the network metrics was similar in all MS subtypes suggesting that these measures could be useful across the whole MS disease spectrum. The same statistical models did not explain SDMT in HC. This negative result is not surprising given the narrow distribution of the SDMT variable in HC compared with patients (see table 2 for mean and SD). Furthermore, due the small number of HC for which we have SDMT $(n=12)$, these results should be interpreted with caution. Future studies could assess whether the findings presented here are replicated in other cohorts.

\section{Descriptive associations among study variables in patients}

Our univariate associations in patients revealed some interesting patterns. Low values of network metrics were associated with clinical impairment and worse information processing speed in accordance with previous studies. ${ }^{822} 25$ Interestingly, reduced clustering showed the strongest association out of network metrics with worse SDMT indicating that network randomisation impairs information processing speed as shown previously. ${ }^{27}$ Our multivariate analysis though demonstrated that reduced network integration and tissue atrophy can more strongly affect SDMT performance. In line with previous work, ${ }^{8}$ WM lesions impair the communication between brain regions at the global and local level as demonstrated by the reduced network efficiencies. As shown in the exact same cohort, we did not find any association between WM lesions and EDSS ${ }^{10}$ and only weak association between WM lesions and SDMT, and this highlights the need to explore non-conventional MRI metrics to explain disability. Also, there was no association between edge density and any of the network efficiencies. Although this could be the result of wiring cost and efficiency, ${ }^{26}$ we argue that direct comparison between binary and weighted network is not valid.

In our approach, we used CSD to model intravoxel crossing fibres ${ }^{20}$ and ACT and SIFT2 to improve connection and streamline density, ${ }^{12} 13$ respectively, with the assumption that the FOD amplitude corresponds to the underlying fibre density. ${ }^{36}$ These advanced methods improve tractogram reconstruction without the need of various scaling techniques. ${ }^{14}$ We also provided anatomical prior of the WM ensuring that no streamlines are incorrectly terminated in the WM due to lesions (online supplementary efigure 1). In fact, we identified an association between LL and connectivity, but this correlation is not that high $(\mathrm{r}=-0.3)$, which highlights that our current approach is not overly influenced by lesions.

\section{Limitations and future directions}

This study has several limitations. In our approach, we applied techniques to address some of the reconstruction biases and to ensure that no streamlines were abnormally terminated in WM. ${ }^{12-14}$ However, histological validation studies are required to make direct links between imaging measures and underlying pathology. Additionally, the cross-sectional design of the study does not allow to determine the clinical relevance of network measures over time. Moreover, we used SDMT scores for approximately half of MS cohort ( $n=60$ vs $n=122$ ), but this subcohort had similar proportions of MS subgroups to the whole cohort (online supplementary etable 1). Also, the effects of fatigue, depression and anxiety can be investigated in future studies with larger cohorts. A post hoc analysis revealed depression and anxiety scores showed mild correlations with SDMT whereas fatigue did not. It is difficult to investigate their influences in our cohort as the HADS and fatigue scores were not collected in all subjects with SDMT. Finally, although the effect of cortical lesions in clinical scores is limited, ${ }^{37}$ it is possible that they may influence our study outcomes.

The study findings could provide the basis for future work. There are different scales that we could study MS from, including micro, meso and macro scales. ${ }^{38}$ Network analysis offers a framework at the macroscale to study whole brain connectivity patterns beyond focal pathology while TBSS, for example, is currently considered a leading technique for the voxel-wise DTI analysis. ${ }^{4}$ Future investigations could focus in the comparison between scales and their link with clinical outcome. Additionally, further studies could follow a subnetwork or nodal rather than global network analysis and perhaps derive integrative measures of structural and functional networks and investigate if these parameters explain additional variance.

\section{CONCLUSION}

In conclusion, we found distinct network organisation in the various groups. Also, network metrics and in particular global efficiency explains disability over and above non-network metrics supporting the relevance of intact long-distance connexions mainly, to maintain normal function. These results highlight the potential of network parameters as biomarkers for disease diagnosis, prognosis and in clinical trials.

\section{Acknowledgements The authors would like to thank all the patients in this study and Professor David Miller and Dr Nils Muhlert for the recruitment of the participants.}

Contributors TC: original design, data analysis, drafting of the manuscript. CT: data analysis, drafting of the manuscript, critical revision. FP: data analysis, critical revision. BK: data analysis, critical revision. DTC: study conception, data acquisition, critical revision. SO: data analysis, critical revision. JDC: original design, data analysis, critical revision. CAMGW-K: original design, data analysis, critical revision. AJT: original design, data analysis, critical revision. ATT: original design, data analysis, critical revision.

Funding The NMR unit where this work was performed is supported by grants from the Multiple Sclerosis Society of Great Britain and Northern Ireland, Philips Healthcare, and supported by the UCL/UCLH NIHR (National Institute for Health Research) BRC (Biomedical Research Centre). TC is supported by grant from the Leonard Wolfson Foundation. CT has received a post-doctoral research ECTRIMS fellowship (2015). FP is supported by a Guarantors of Brain non-clinical postdoctoral fellowship. SO is funded by the National Institute for Health Research University College London Hospitals Biomedical Research Centre (NIHR BRC UCLH/UCL High Impact Initiative BW.mn.BRC10269). SO also receives funding from the EPSRC (EP/ 
H046410/1, EP/J020990/1, EP/K005278), the MRC (MR/J01107X/1) and the NIHR Biomedical Research Unit (Dementia) at UCL. CAMGW-K has received research grants (PI and co-applicant) from Spinal Research, Craig H. Neilsen Foundation, EPSRC, Wings for Life, UK MS Society, Horizon2020, NIHR/MRC.

Competing interests AJT has received honoraria/support for travel for consultancy from Eisai, Biogen (Optum Insight), Hoffman La Roche, MedDay, TEVA, Almirall, and Excemed. He received support for travel for consultancy as chair of the International Progressive MS Alliance Scientific Steering Committee, and member of the National MS Society (USA) Research Programs Advisory Committee. He receives an honorarium from SAGE Publishers as Editor-in-Chief of Multiple Sclerosis Journal and a free subscription from Elsevier as a board member for the Lancet Neurology. Support from the NIHR UCLH Biomedical Research Centre is acknowledged. CT has received an ECTRIMS post-doctoral research fellowship in 2015. She has also received honoraria and support for travelling from BayerSchering, Teva, Merck-Serono and Serono Foundation, Biogen, Sanofi-Aventis, Novartis, and Ismar Healthcare. FP is non-clinical Guarantors of the Brain fellow. He has also received honoraria from Bioclinica Inc. DC has received honoraria (paid to his employer) from Ismar Healthcare NV, Swiss MS Society, Excemed (previously Serono Symposia International Foundation), Merck, Bayer and Teva for faculty-led education work; Teva for advisory board work; meeting expenses from Merck, MS Trust, National MS Society, Novartis, Société des Neurosciences and Teva; and has previously held stock in GlaxoSmithKline. ATT has received speaker honoraria from Biomedia, Sereno Symposia International Foundation, Bayer and meeting expenses from Biogen Idec and is the UK PI for two clinical trials sponsored by MEDDAY pharmaceutical company (MD1003 in optic neuropathy [MS-ON] and progressive MS [MS-SPI2]).

Patient consent Obtained.

Ethics approval This study was approved by the local UCL Research Ethics Committee.

Provenance and peer review Not commissioned; externally peer reviewed.

Open access This is an open access article distributed in accordance with the Creative Commons Attribution 4.0 Unported (CC BY 4.0) license, which permits others to copy, redistribute, remix, transform and build upon this work for any purpose, provided the original work is properly cited, a link to the licence is given, and indication of whether changes were made. See: http://creativecommons.org/ licenses/by/4.0

\section{REFERENCES}

1. Mahad DH, Trapp BD, Lassmann H. Pathological mechanisms in progressive multiple sclerosis. Lancet Neurol 2015;14:183-93.

2. Fisniku LK, Brex PA, Altmann DR, et al. Disability and T2 MRI lesions: a 20-year followup of patients with relapse onset of multiple sclerosis. Brain 2008;131(Pt 3):808-17.

3. Muhlert N, Sethi V, Cipolotti L, et al. The grey matter correlates of impaired decisionmaking in multiple sclerosis. J Neurol Neurosurg Psychiatry 2015;86:530-6.

4. Dineen RA, Vilisaar J, Hlinka J, et al. Disconnection as a mechanism for cognitive dysfunction in multiple sclerosis. Brain 2009;132(Pt 1):239-49.

5. Rocca MA, Amato MP, De Stefano N, et al. Clinical and imaging assessment of cognitive dysfunction in multiple sclerosis. Lancet Neurol 2015;14:302-17.

6. Kurtzke JF. Rating neurologic impairment in multiple sclerosis: an expanded disability status scale (EDSS). Neurology 1983;33:1444-52.

7. Fornito A, Zalesky A, Breakspear M. The connectomics of brain disorders. Nat Rev Neurosci 2015;16:159-72.

8. Shu N, Liu Y, Li K, et al. Diffusion tensor tractography reveals disrupted topological efficiency in white matter structural networks in multiple sclerosis. Cereb Cortex 2011;21:2565-77.

9. Fleischer V, Gröger A, Koirala N, et al. Increased structural white and grey matter network connectivity compensates for functional decline in early multiple sclerosis. Mult Scler 2017;23:432-41.

10. Pardini $M$, Yaldizli Ö, Sethi $V$, et al. Motor network efficiency and disability in multiple sclerosis. Neurology 2015;85:1115-22.

11. Sotiropoulos SN, Zalesky A. Building connectomes using diffusion MRI: why, how and but. NMR Biomed 2017:e3752.

12. Smith RE, Tournier JD, Calamante F, et al. Anatomically-constrained tractography: improved diffusion MRI streamlines tractography through effective use of anatomical information. Neuroimage 2012;62:1924-38.
13. Smith RE, Tournier JD, Calamante F, et al. SIFT2: enabling dense quantitative assessment of brain white matter connectivity using streamlines tractography. Neuroimage 2015;119:338-51.

14. Yeh CH, Smith RE, Liang X, et al. Correction for diffusion MRI fibre tracking biases: the consequences for structural connectomic metrics. Neuroimage 2016;142:150-62.

15. Lublin FD, Reingold SC. Defining the clinical course of multiple sclerosis: results of an international survey. National Multiple Sclerosis Society (USA) advisory committee on clinical trials of new agents in multiple sclerosis. Neurology 1996;46:907-11.

16. Bhushan C. Correcting susceptibility-induced distortion in diffusion-weighted MRI using constrained nonrigid registration. Signal Inf Process Assoc Annu Summit Conf APSIPA Asia Pac, 2012.

17. Prados F, Cardoso MJ, Kanber B, et al. A multi-time-point modality-agnostic patchbased method for lesion filling in multiple sclerosis. Neuroimage 2016;139:376-84

18. Cardoso MJ, Modat M, Wolz R, et al. Geodesic information flows: spatially-variant graphs and their application to segmentation and fusion. IEEE Trans Med Imaging 2015;34:1976-88.

19. Andersson JLR, Sotiropoulos SN. An integrated approach to correction for offresonance effects and subject movement in diffusion MR imaging. Neuroimage 2016:125:1063-78.

20. Tournier JD, Calamante F, Connelly A. Robust determination of the fibre orientation distribution in diffusion MRI: non-negativity constrained super-resolved spherical deconvolution. Neuroimage 2007;35:1459-72.

21. Kocevar G, Stamile C, Hannoun S, et al. Graph theory-based brain connectivity for automatic classification of multiple sclerosis clinical courses. Front Neurosci 2016;10:478.

22. Shu N, Duan Y, Xia M, et al. Disrupted topological organization of structural and functional brain connectomes in clinically isolated syndrome and multiple sclerosis. $\mathrm{SCl}$ Rep 2016;6:29383.

23. Rocca MA, Valsasina P, Meani A, et al. Impaired functional integration in multiple sclerosis: a graph theory study. Brain Struct Funct 2016;221:115-31.

24. Liu Y. Functional brain network alterations in clinically isolated syndrome and multiple sclerosis: a graph-based connectome study. Radiology 2017;282:534-41.

25. Llufriu S, Martinez-Heras E, Solana E, et al. Structural networks involved in attention and executive functions in multiple sclerosis. Neuroimage Clin 2017;13:288-96.

26. Bullmore E, Sporns 0 . The economy of brain network organization. Nat Rev Neurosci 2012;13:336-49.

27. Douw L, Schoonheim MM, Landi D, et al. Cognition is related to resting-state small-world network topology: an magnetoencephalographic study. Neuroscience 2011:175:169-77.

28. Muthuraman M, Fleischer $V$, Kolber $P$, et al. Structural brain network characteristics can differentiate CIS from early RRMS. Front Neurosci 2016;10:14.

29. Tewarie P, Steenwijk MD, Tijms BM, et al. Disruption of structural and functional networks in long-standing multiple sclerosis. Hum Brain Mapp 2014;35:5946-61.

30. Schoonheim MM, Hulst HE, Landi D, et al. Gender-related differences in functional connectivity in multiple sclerosis. Mult Scler 2012;18:164-73.

31. Friese MA, Schattling B, Fugger L. Mechanisms of neurodegeneration and axonal dysfunction in multiple sclerosis. Nat Rev Neurol 2014;10:225-38.

32. Mangeat $G$, Badji $A$, Ouellette $R$, et al. Changes in structural network are associated with cortical demyelination in early multiple sclerosis. Hum Brain Mapp 2018;39:2133-46.

33. Tewarie P, Schoonheim MM, Schouten DI, et al. Functional brain networks: linking thalamic atrophy to clinical disability in multiple sclerosis, a multimodal fMRI and MEG study. Hum Brain Mapp 2015;36:603-18.

34. Schoonheim MM, Meijer KA, Geurts JJ. Network collapse and cognitive impairment in multiple sclerosis. Front Neurol 2015;6: :82.

35. Tuladhar AM, van Uden IW, Rutten-Jacobs LC, et al. Structural network efficiency predicts conversion to dementia. Neurology 2016;86:1112-9.

36. Raffelt D, Tournier JD, Rose S, et al. Apparent Fibre Density: a novel measure for the analysis of diffusion-weighted magnetic resonance images. Neuroimage 2012;59:3976-94.

37. van de Pavert SH, Muhlert N, Sethi V, et al. DIR-visible grey matter lesions and atrophy in multiple sclerosis: partners in crime? I Neurol Neurosurg Psychiatry 2016:87:461-7.

38. Cercignani M, Gandini Wheeler-Kingshott C. From micro- to macro-structures in multiple sclerosis: what is the added value of diffusion imaging. NMR Biomed 2018:e3888

39. Clayden J. TractoR: magnetic resonance imaging and tractography with R. JSS, 2011.

40. Rubinov M, Sporns 0 . Complex network measures of brain connectivity: uses and interpretations. Neuroimage 2010;52:1059-69. 